\title{
Artroplastia de suspensión dinámica de Scheker en el tratamiento de la artrosis trapeciometacarpiana
}

\section{Scheker Dynamic Arthroplasty in the Treatment of Trapeziometacarpal Arthrosis}

\author{
C. D. Novoa-Parra ${ }^{1} \quad$ J. I. Pérez-Correa ${ }^{1} \quad$ S. Pérez-Ortiz ${ }^{1} \quad$ D. Montaner-Alonso ${ }^{1,2} \quad$ J. L. Rodrigo-Pérez ${ }^{1,2}$ \\ M. Morales-Suarez-Varela2,3,4
}

${ }^{1}$ Servicio de Cirugía Ortopédica y Traumatología, Hospital

Address for correspondence Carlos Daniel Novoa-Parra, MD, Servicio Universitario Dr. Peset, Valencia, España

2 Universidad de Valencia, Valencia, España

3 Departamento de Medicina Preventiva, Universidad de Valencia, Dr. Peset, Dirección postal: Av Profesor López Piñero Nํ12, Piso 7 , Valencia, España

${ }^{4}$ CIBER Epidemiología y Salud Pública (CIBERESP), Instituto de Salud Carlos III, Madrid, España

Puerta 26, Valencia, España (e-mail: le_male2002@hotmail.com).

Rev Iberam Cir Mano 2017;45:115-119.

\section{Resumen \\ Palabras Clave \\ - artrosis carpometacarpiana \\ - reconstrucción del ligamento \\ - artroplastia de interposición}

Abstract
Objetivo Evaluar los resultados clínicos y radiográficos de una serie de pacientes sometidos a una artroplastia de Scheker.

Material y Método Estudio descriptivo de 16 pacientes con artrosis TMC. Se valoró el estado clínico y funcional según el cuestionario DASH, la movilidad del pulgar según la escala de Kapandji y el dolor según la escala visual analógica (EVA). A nivel radiográfico, se cuantificó el colapso del espacio remanente tras la exéresis del trapecio.

Resultados La edad promedio de los pacientes fue 64,9 años, $81 \%$ fueron mujeres. El tiempo de seguimiento medio fue de 19 meses. La puntuación DASH preoperatoria fue de 67,68 puntos y postoperatoria $34,88(p<0.001)$. La puntuación EVA preoperatoria fue 8,44 y posoperatoria 1,69 , $(p<0.001)$. La movilidad media del pulgar según Kapandji fue 7, $(p<0.001)$. Las fuerzas de puño, pinza de precisión y pinza lateral, pasaron de valores medios de 11,94, 3,41 y 3,46 en el preoperatorio, a valores medios de 1,25, 2,44 y 2,34 en el postoperatorio. En todos los casos se observó un cierto grado de colapso (31,38\% de promedio) del espacio ocupado por el trapecio. El 87,5\% de los pacientes se encontraban satisfechos con la cirugía y volverían a someterse a la misma. Conclusión La artroplastia descrita por Scheker, es una alternativa quirúrgica que elimina el dolor y preserva la movilidad del pulgar. No evita el colapso del espacio dejado tras la exéresis del trapecio. No hubo diferencia en el resultado clínico entre los pacientes en los que se presentó un colapso leve o moderado.

Purpose To evaluate the clinical and radiographic results of a series of patients undergoing a Scheker arthroplasty. received

May 23, 2017

accepted

August 31, 2017

published online

September 25, 2017
DOI https://doi.org/

10.1055/s-0037-1607045.

ISSN 1698-8396.
Copyright @ $\odot 2017$ Thieme Revinter

Publicações Ltda, Rio de Janeiro, Brazil
License terms

(ㄷ) (i) $\ominus$ (\$) 
Methods Descriptive study of 16 patients with trapeziometacarpal arthrosis. Clinical and functional status according to the DASH questionnaire, thumb mobility according to the Kapandji scale and visual analogue scale (VAS) were assessed. At the radiographic collapse of the remaining space was quantified after the trapezium excision.

Results Mean age of the patients was 64.9 years, $81 \%$ were women. Mean follow-up was 19 months. Preoperative DASH score was 67.68 points and postoperative 34.88 $(p<0.001)$. Preoperative VAS score was 8.44 and postoperative $1.69(p<0.001)$. Mean mobility of the thumb according to Kapandji was 7 , $(p<0.001)$. Forces $(\mathrm{Kg})$ of the fist, tip pinch and key pinch, went from $11.94,3.41$ and 3.46 preoperative to 1.25 , 2.44 and 2.34 in the postoperative period. In all cases a certain degree of collapse ( $31.38 \%$ of average) was observed. $87.5 \%$ of the patients were satisfied with the surgery and would undergo it again.

Conclusion Arthroplasty described by Scheker is a surgical alternative that eliminates the pain and preserves the mobility of the thumb. It does not prevent the collapse of the space left after the excision of the trapezium. There was no difference in clinical outcome among patients in whom mild or moderate collapse occurred.

\section{Introducción}

Las opciones quirúrgicas para el tratamiento de la artrosis trapeciometacarpiana (TMC) incluyen: trapecectomía, trapecectomía combinada con interposición tendinosa, artrodesis, artroplastia de implante o de sustitución, y trapecectomía más reconstrucción e interposición tendinosa (RLIT). De todas ellas, la más comúnmente utilizada es la RLIT, la cual fue introducida en 1986 por Burton y Pellegrini. ${ }^{1}$ Desde entonces, se han descrito numerosas modificaciones de la misma, ${ }^{2}$ entre las que se encuentra la de Scheker, ${ }^{3}$ que permite suspender el primer metacarpiano mediante la utilización de un hemitendón del flexor carpi radialis (FCR), al mismo tiempo que reconstruye simultáneamente los ligamentos intermetacarpiano y oblicuo anterior, respectivamente.

El objetivo de este trabajo es evaluar los resultados clínicos-funcionales y el grado de satisfacción personal, de una serie de pacientes con artrosis TMC a los que se les intervino quirúrgicamente mediante el procedimiento descrito por Scheker. ${ }^{3}$ Asimismo y dentro de la misma serie, se compararon dos grupos de pacientes teniendo en cuenta el grado de colapso del espacio remanente tras la exéresis del trapecio.

\section{Material y Método}

Se llevó a cabo un estudio de cohortes de pacientes con rizartrosis intervenidos entre 2014 y 2016 mediante la técnica de Scheker. En todos los pacientes se había intentado el tratamiento conservador previamente, incluyendo la inmovilización, antiinflamatorios no esteroideos (AINEs) e infiltraciones articulares con corticoesteroides, sin respuesta.

Las radiografías para la valoración de rizartrosis fueron en proyección postero-anterior; proyección lateral y proyección oblicua de la mano centrada en la TMC. Para clasificar la severidad radiológica en el momento del diagnóstico se utilizó la clasificación de Eaton-Glickel, ${ }^{4}$ la cual es independiente de los datos clínicos subjetivos y objetivos, y se basa únicamente en los hallazgos radiográficos.

En el protocolo de nuestro hospital, los pacientes son examinados clínicamente a las 3 y 6 semanas, y a los 3, 6 y 12 meses de la cirugía. El seguimiento radiográfico se hace a los 3, 6 y 12 meses. Se seleccionaron los casos con por lo menos 6 meses de seguimiento.

La muestra estuvo representada por 16 pacientes, siendo 13 mujeres y 3 hombres, con una edad media de 65 (rango: 53 a 81) años. 8 manos intervenidas fueron derechas y 8 izquierdas. El seguimiento promedio fue de 19 (Rango: 6 a 31) meses. La ocupación más frecuente fue ama de casa (56.25\%), seguida por pacientes que ya no se encontraban en edad laboral (31.25\%) y trabajadores manuales (12.5\%)

En la valoración previa a la cirugía, 10 pacientes se encontraban en un estadio III y 6 en un estadio IV, según la clasificación de Eaton-Glickel.

Se registraron las complicaciones relacionadas con la cirugía y el tiempo de seguimiento. El resultado se valoró con medidas preoperatorias y postoperatorias a través del cuestionario $\mathrm{DASH}^{5}$ la movilidad según la escala de Kapandji 6 y el dolor según la escala visual analógica (EVA). Para la medición fuerza de pinza punta-punta y pinza lateral, así como la fuerza de puño antes y después de la intervención se usaron dinamómetros Jamar ${ }^{\circledR}$ (Asimov Engineering, Los Ángeles, CA). Para valorar el grado de satisfacción del paciente incluimos como preguntas: « ¿se encuentra satisfecho con la cirugía?» y "¿volvería a operarse?».

El colapso del espacio tras la artroplastia fue valorado en la radiografía PA a los 6 meses. Para ello, la altura del espacio de la artroplastia se normalizó para la ampliación de los rayos $\mathrm{X}$ mediante su relación proporcional a la longitud del primer metacarpiano (índice $=$ altura del espacio de la artroplastia / longitud del primer metacarpiano). Junto con esa medida, se calculó el cociente antes/después de la cirugía, que se expresó como porcentaje de colapso. ${ }^{7}$ De acuerdo a dicho colapso, se 
graduó el mismo en: leve (0-33\%) del espacio de artroplastia inicial, moderado (34-66\%) y grave (67-100\%).

El análisis de los datos se realizó con el paquete estadístico para las ciencias sociales (SPSS) versión 21.0, considerando en todos los casos un nivel de significación estadística del 95\% $(p<0.05)$. Para las variables cuantitativas, los resultados descriptivos se expresaron mediante media y desviación estándar. Las variables cualitativas se expresaron mediante número y porcentaje. La comparación de medias se realizó mediante $t$-test.

\section{Técnica Quirúrgica}

El procedimiento descrito por $\operatorname{Scheker}^{3}$ ( - Fig. 1) se realiza bajo anestesia regional e isquemia del miembro mediante un manguito de presión colocado a nivel del brazo. Finalizado el procedimiento, se comprueba que el primer metacarpiano no se hunde en el espacio dejado tras la trapecectomía y que se ha conseguido la apertura del primer espacio interdigital. La capsula articular se cierra con sutura reabsorbible $3 / 0$ y los márgenes de la piel se cierran utilizando Nylon 4/0. El pulgar del paciente se coloca en abducción y se inmoviliza durante 3 semanas con una férula de yeso volar que incluye la articulación interfalángica. A partir de la tercera semana, se retira la férula y el paciente comienza la rehabilitación mediante movilización progresiva. Los ejercicios de fortalecimiento de pinza y presa se inician a partir de la 6 semana.

\section{Resultados}

En todos los pacientes el nivel de dolor calculado según EVA se redujo significativamente de 8,43 puntos a 1,69 puntos $(p=0,001)$.

La fuerza de puño disminuyó de $11,94 \mathrm{Kg}$ prequirúrgica para $11,25 \mathrm{Kg}$ posquirúrgica; la fuerza de pinza de precisión (Tip-to-tip) disminuyo de 3,41 $\mathrm{Kg}$ para $2,44 \mathrm{Kg}$; la fuerza de pinza lateral (Key) disminuyó de 3,46 $\mathrm{Kg}$ para 2,34 Kg después de la cirugía, aunque sin significación estadística.

El rango de movilidad, según la escala de Kapandji, mejoró desde la posición 5 a la posición 7 después de la cirugía $(p=0,001)$.

Al valorar la funcionalidad del miembro intervenido mediante la escala DASH se obtuvo un resultado favorable, pasando de 67,68 a 34,88 puntos ( $p=0,001$ )

Para el grado de colapso radiológico tras la exéresis del trapecio, se obtuvo un valor promedio del 31,38\%. El hundimiento o colapso fue leve en 8 pacientes y moderado en el resto. No se encontró ningún paciente con colapso severo ( $\geq 67 \%$ del espacio de la artroplastia) (Tabla 1).

Con respecto a ese último parámetro, comparamos los resultados posquirúrgicos dividiendo la muestra en dos grupos dependiendo del colapso del espacio de la artroplastia. En el grupo que presentó colapso leve ( $\leq 33 \% \mathrm{del}$ espacio de la artroplastia) el EVA promedio fue de 1,8 puntos; la fuerza promedio fue: puño 12,8 Kg, pinza de precisión 2,58 Kg, pinza lateral 2,43 Kg; movilidad según la escala de Kapandji 7; DASH 34,5 puntos. En el grupo que presentó un colapso moderado (34-66\% del espacio de la artroplastia), el EVA promedio fue de 1,5 puntos; la fuerza: de puño $9,65 \mathrm{Kg}$, pinza de precisión 2,3 $\mathrm{Kg}$, pinza lateral 2,23 Kg; movilidad según Kapandji 7; DASH 35,25. No se encontraron diferencias significativas entre las variables estudiadas de ambos grupos (Tabla 2).

El 87,5\% (14) de los pacientes se encontraban satisfechos con la cirugía y volverían a someterse a la misma. El 12,5\% (2) de los pacientes no se encontraban satisfechos con el resultado y no se volverían a operar. Uno de ellos, con colapso moderado y en edad laboral, se encontraba descontento por la pérdida de fuerza a pesar de la mejoría del dolor previo. El otro fue un posoperatorio complicado, pues sufrió una caída que le causó una inmovilización muy

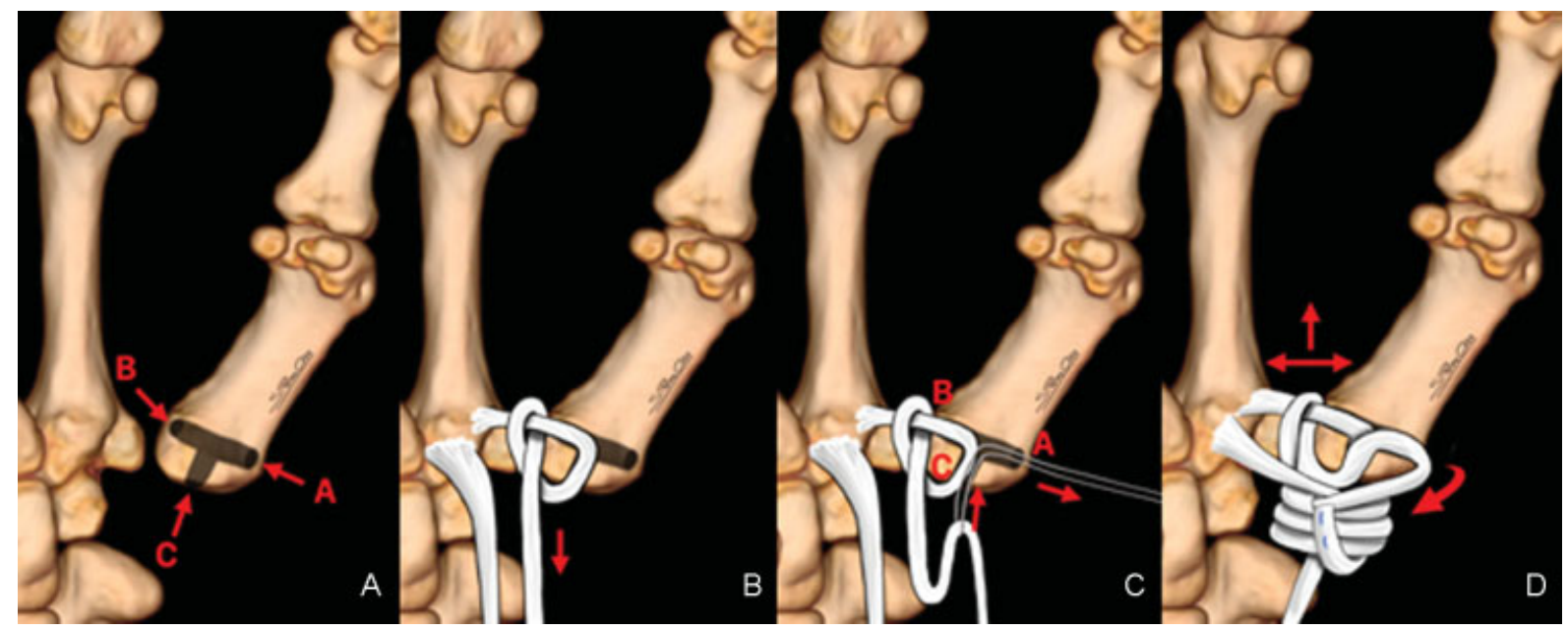

Fig. 1 Visión palmar, posterior a la resección de trapecio. (A): Dos túneles que se intersectan para formar una forma de “T.” El túnel $A B$ se extiende desde el lado dorso-radial del metacarpiano hasta el lado ulno-palmar del metacarpiano. El túnel C que se extiende perpendicular al túnel AB. (B): El hemitendón del FCR (Flexor carpi radialis), pasa por el túnel B, sale por el túnel Cy se realiza un bucle alrededor del hemitendón que forma un espaciador entre las bases de los dos metacarpianos. (C) El hemitendón se pasa nuevamente por el túnel Cy sale por el túnel A. (D) El hemitendón se envuelve alrededor de la mitad anclada al segundo metacarpiano, se crea una anchoa que sirve como espaciador entre la base del primer metacarpiano y el polo distal del escafoides. La flecha representa el vector de fuerza que se crea al traccionar del hemitendón, y con eso se logra la apertura del primer espacio interdigital y la pronación del primer metacarpiano. Los tendones son suturados con Nylon 4/0. 
Tabla 1 Resultados clínicos y funcionales después de la plastia de Scheker

\begin{tabular}{|l|l|l|l|l|l|l|}
\hline \multicolumn{2}{|l|}{ EVA } & Puño $(\mathrm{Kg})$ & PP $(\mathrm{Kg})$ & PL $(\mathrm{Kg})$ & Kapandji & DASH \\
\hline Pre & $8,44 \pm 0,89$ & $11,94 \pm 5,20$ & $3,41 \pm 1,81$ & $3,46 \pm 1,99$ & $5,06 \pm 0,25$ & $67,68 \pm 4,69$ \\
\hline Post & $1,69 \pm 1,34$ & $11,25 \pm 5,10$ & $2,44 \pm 2,03$ & $2,34 \pm 1,87$ & $7,06 \pm 0,25$ & $34,88 \pm 2,96$ \\
\hline$p$ valor & 0,001 & 0,377 & 0,163 & 0,109 & 0,001 & 0,001 \\
\hline
\end{tabular}

Abreviaciones: EVA, escala visual analógica; PL, fuerza de pinza punta-lateral; PP, fuerza de pinza punta-punta.

Media \pm Desviación estándar. $t$-test

Tabla 2 Resultados clínicos y funcionales después de la plastia de Scheker dividiendo la muestra según el grado de colapso de la artroplastia

\begin{tabular}{|l|l|l|l|l|l|l|}
\hline EVA & Puño $(\mathrm{Kg})$ & PP $($ Kg) & PL $($ Kg) & Kapandji & DASH \\
\hline Leve & $1,88 \pm 1,45$ & $12,88 \pm 6,57$ & $2,58 \pm 2,23$ & $2,43 \pm 1,89$ & $7,13 \pm 0,35$ & $34,5 \pm 1,32$ \\
\hline Moderado & $1,50 \pm 0,46$ & $9,63 \pm 2,56$ & $2,30 \pm 1,95$ & $2,23 \pm 1,97$ & $7,00 \pm 0,01$ & $35,25 \pm 0,75$ \\
\hline$p$ valor & 0,529 & 0,214 & 0,788 & 0,839 & 0,334 & 0,632 \\
\hline
\end{tabular}

Abreviaciones: EVA, escala visual analógica; PL, fuerza de pinza punta-lateral; PP, fuerza de pinza punta-punta.

Media \pm Desviación estándar. $t$-test

prolongada que afectó el proceso de rehabilitación. Ese paciente presentó un colapso leve de la artroplastia. No se encontraron otras complicaciones durante el seguimiento.

\section{Discusión}

Desde que Burton y Pellegrini ${ }^{1}$ describieron en 1986 la primera técnica de RLIT, se han descrito modificaciones de ella, aunque ninguna añade el gesto de interposición de tejido (tendinoso) entre las bases del primer y segundo metacarpianos, con el fin de evitar el dolor causado por el contacto de ellas. Sin embargo, la técnica descrita por Scheker si lo hace, además de reconstruir el ligamento intermetacarpiano, encargado de evitar la migración proximal del primer metacarpiano tras la resección del trapecio. No obstante, a pesar de todas esas teóricas ventajas, en nuestra serie se produjo en todos los casos un colapso parcial, leve o moderado, aunque sin diferencia clínica entre ambos grupos. Muchos estudios no han encontrado una asociación entre el mantenimiento del espacio dejado tras la exéresis del trapecio y la fuerza, alivio del dolor o función del pulgar. ${ }^{8}$

Pese a todos los esfuerzos que los cirujanos realizan para evitar el colapso de la artroplastia, algunos argumentan varias razones por las que es muy difícil de prevenir, entre las que cabe citar la imposibilidad de rellenar todo el defecto dejado tras la resección del trapecio con solo una bandeleta del FCR. Incluso utilizando todo el espesor del mismo, tampoco se evita cierto grado de colapso. ${ }^{9-11}$ De hecho, el mismo Scheker, en su técnica original, sugirió el relleno de los túneles óseos con fragmentos óseos provenientes del mismo trapecio resecado, para aumentar la tensión del tendón, así como mantener la inmovilización durante 6 semanas, momento en el cual se inicia la rehabilitación y se mantiene al paciente con férula de protección para las actividades de más demanda funcional durante 12 semanas; todo ello, con el fin de lograr una mejor integración tendón-hueso y prevenir así, la migración proximal del primer metacarpiano.
En cuanto al dolor, en nuestra serie, disminuyó de forma significativa pasando de 8,3 puntos preoperatorios a 1,68 puntos posoperatorios, lo cual es concordante con la bibliografía previa (Tabla 3 ).

En relación a la fuerza de puño, pinza de precisión y pinza lateral ellas disminuyeron después del procedimiento, aunque no de forma estadísticamente significativa. Ese hecho se explica por el poco tiempo de evolución; la bibliografía al respecto, indica que se necesita al menos 6 años para equipararse con las medidas preoperatorias. Incluso, solo el $34 \%$ de los pacientes recuperan una fuerza normal. ${ }^{12}$

En referencia al movimiento, nuestros pacientes mejoraron la oposición del pulgar según el esquema de Kapandji. Uno de los problemas en la artrosis TMC avanzada, es la limitación de ese movimiento, por lo que consideramos su medida de particular interés al valorar la evolución de los pacientes. Sin embargo, si comparamos nuestros resultados con la bibliografía publicada, la oposición promedio de nuestros pacientes fue significativamente menor que la media de las series publicadas (Tabla $\mathbf{3}$ ).

Por último, el $87,5 \%$ de los pacientes, se encontraban satisfechos con la cirugía y volverían a someterse a la misma, cifras muy similares a las publicadas con este tipo de procedimientos (85\% a $100 \%){ }^{8}$

La limitación de este trabajo es que se trata de una serie de casos y que el número y tiempo de seguimiento de los pacientes es muy corto para sacar conclusiones definitivas. Sin embargo, la fortaleza del mismo radica en que al ser un estudio unicéntrico, la validez interna es mayor, no solo por la homogeneidad en el protocolo de tratamiento sino también en la uniformidad en la valoración de pruebas.

\section{Conclusiones}

La plastia de reconstrucción ligamentosa con interposición tendinosa (RLIT) descrita por Scheker, mejora de manera 
Tabla 3 Series de casos de artroplastias tipo RLIT (reconstrucción ligamentaria con interposición tendinosa) para el tratamiento de la rizartrosis en estadios avanzados

\begin{tabular}{|c|c|c|c|c|c|c|c|c|c|c|}
\hline Estudio (año) & $\begin{array}{l}\text { Procedimiento } \\
\text { RLIT }\end{array}$ & Muestra & $\begin{array}{l}\text { Media } \\
\text { de edad } \\
\text { (años) }\end{array}$ & $\begin{array}{l}\text { Seguimiento } \\
\text { años }\end{array}$ & $\begin{array}{l}\text { ABD } \\
\text { radial } \\
\left({ }^{\circ}\right)\end{array}$ & $\begin{array}{l}\text { ABD } \\
\text { palmar } \\
\left({ }^{\circ}\right)\end{array}$ & $\begin{array}{l}\text { Oposición } \\
\text { del pulgar } \\
\text { (Kapandji) }\end{array}$ & $\begin{array}{l}\text { Puño } \\
\text { (Kg) }\end{array}$ & $\begin{array}{l}\text { Pinza } \\
\text { PP (Kg) }\end{array}$ & $\begin{array}{l}\text { Alivio del } \\
\text { dolor EVA } \\
\text { (puntos) }\end{array}$ \\
\hline Varitimidis y col. ${ }^{11}(2000)$ & FCR entero & 62 & 58,4 & 3,5 & 44 & 41 & - & 22 & 4,6 & - \\
\hline De Semet y col. ${ }^{13}(2002)$ & Burton-Pellegrini & 31 & 57 & 2,1 & - & - & 9,3 & 26 & - & 2,5 \\
\hline Scheker y col. ${ }^{3}(2004)$ & Scheker & 238 & 55,2 & 1,92 & - & - & 9,3 & 22,08 & - & 1,6 \\
\hline Basar y col. ${ }^{10}$ (2012) & FCR entero & 19 & 55 & 4,91 & 38 & - & 9,5 & 19,23 & 4,45 & 1,4 \\
\hline Yang y col. ${ }^{14}(2014)$ & Scheker & 21 & 60 & 1,15 & 60,6 & 63,5 & - & 18,6 & 4,4 & 0,5 \\
\hline Merenghi y col. ${ }^{15}$ (2016) & FCR entero & 100 & 62,4 & 3 & - & - & 9,4 & 21,6 & 4,1 & 1,5 \\
\hline Media de estudios previos & - & $n=471$ & 57,33 & 2,82 & - & - & 9,3 & 21,58 & 4,38 & 1,5 \\
\hline Estudio actual. (2017) & Scheker & 16 & 64,9 & 1,58 & - & - & 7,06 & 11,25 & 2,4 & 1,6 \\
\hline$p$ valor & - & - & 0,088 & 0,197 & - & - & 0,001 & 0,001 & 0,067 & 0,876 \\
\hline
\end{tabular}

Abreviaciones: $\left({ }^{\circ}\right)$, grados; ABD, abducción; DASH, cuestionario Disabilities of the Arm, Shoulder and Hand; EVA, escala visual analógica del dolor; PL, punta-lateral; PP, punta-punta.

Para el cálculo de la media se tomaron las variables que tuviesen por lo menos 4 valores.

significativa el dolor, la oposición del pulgar y probablemente todo su rango de movimiento, pero en nuestra serie no mejora la fuerza, ni es efectiva para prevenir del todo la migración proximal del primer metacarpiano. No hubo diferencia en el resultado clínico entre los pacientes en los que se presentó un colapso leve o moderado.

\section{Protección de Personas y Animales}

El autor declara que para esta investigación no se han realizado experimentos en seres humanos ni en animales.

\section{Confidencialidad de los Datos}

El autor declara que en este artículo no aparecen datos de pacientes.

Derecho a la Privacidad y Consentimiento Informado El autor declara que en este artículo no aparecen datos de pacientes.

\section{Conflicto de Intereses}

El autor declara que no ha recibido ayuda económica alguna para la realización de este trabajo. Tampoco ha firmado ningún acuerdo por el que vayan a recibir beneficios u honorarios por parte de alguna entidad comercial. Por otra parte, ninguna entidad comercial ha pagado ni pagará a fundaciones, instituciones educativas u otras organizaciones sin ánimo de lucro a las que el autor esté afiliado.

\section{Bibliografía}

1 Burton RI, Pellegrini VD Jr. Surgical management of basal joint arthritis of the thumb. Part II. Ligament reconstruction with tendon interposition arthroplasty. J Hand Surg Am 1986;11(03):324-332

2 Vermeulen GM, Slijper H, Feitz R, Hovius SE, Moojen TM, Selles RW. Surgical management of primary thumb carpometacarpal osteoarthritis: a systematic review. J Hand Surg Am 2011;36(01): 157-169
3 Scheker LR, Boland MR. Dynamic suspension-sling arthroplasty with intermetacarpal ligament reconstruction for the treatment of trapeziometacarpal osteoarthritis. Eur J Plast Surg 2004; 27:185-193

4 Eaton RG, Glickel SZ. Trapeziometacarpal osteoarthritis. Staging as a rationale for treatment. Hand Clin 1987;3(04):455-471

5 Hervás MT, Navarro Collado MJ, Peiró S, Rodrigo Pérez JL, López Matéu P, Martínez Tello I. Versión española del cuestionario DASH. Adaptación transcultural, fiabilidad, validez y sensibilidad a los cambios. Med Clin (Barc) 2006;127(12):441-447

6 Kapandji A. [Clinical test of apposition and counter-apposition of the thumb]. Ann Chir Main 1986;5(01):67-73

7 Yang SS, Weiland AJ. First metacarpal subsidence during pinch after ligament reconstruction and tendon interposition basal joint arthroplasty of the thumb. J Hand Surg Am 1998;23(05):879-883

8 Haase SC, Chung KC. An evidence-based approach to treating thumb carpometacarpal joint arthritis. Plast Reconstr Surg 2011; 127(02):918-925

9 ElfarJC, Burton RI. Ligament reconstruction and tendon interposition for thumb basal arthritis. Hand Clin 2013;29(01):15-25

10 Başar H, Başar B, Erol B, Tetik C. Ligament reconstruction and tendon interposition arthroplasty of the trapeziometacarpal joint with the use of the full thickness of the flexor carpi radialis tendon. Chir Main 2012;31(06):331-336

11 Varitimidis SE, Fox RJ, King JA, Taras J, Sotereanos DG. Trapeziometacarpal arthroplasty using the entire flexor carpi radialis tendon. Clin Orthop Relat Res 2000;(370):164-170

12 Tomaino MM, Pellegrini VD Jr, Burton RI. Arthroplasty of the basal joint of the thumb. Long-term follow-up after ligament reconstruction with tendon interposition. J Bone Joint Surg Am 1995;77(03):346-355

13 De Smet L, Vanfleteren L, Sioen W, Spaepen D, Van Ransbeeck H. Ligament reconstruction/tendon interposition arthroplasty for thumb basal joint osteoarthritis preliminary results of a prospective outcome study. Acta Orthop Belg 2002;68(01):20-23

14 Yang Y, Tien HY, Kumar KK, et al. Ligament reconstruction with tendon interposition arthroplasty for first carpometacarpal joint osteoarthritis. Chin Med J (Engl) 2014;127(22):3921-3925

15 Marenghi L, Paterlini M, Tocco S, Corradi M. Trapeziectomy with ligament reconstruction and tendon interposition arthroplasty with the entire width of the flexor carpi radialis tendon. Tech Hand Up Extrem Surg 2016;20(02):67-70 